\title{
APLICAÇÃO DA TEORIA GREY E FMEA - ANÁLISE DOS MODOS DE FALHA E EFEITOS NA PRIORIZAÇÃO DE RISCOS DE PROJETOS DE DESENVOLVIMENTO DE SOFTWARE PRODUTO
}

\section{APPLICATION OF GRAY THEORY AND FMEA - FAILURE MODES AND EFFECTS ANALYSIS IN RISK PRIORITIZATION DURING SOFTWARE PRODUCT DEVELOPMENT}

\author{
Roberto Carlos Bonanomi ${ }^{1}$; Wesley Vieira da Silva ${ }^{2}$; Jansen Maia Del Corso ${ }^{3}$; Luiz Carlos Duclós ${ }^{4}$ \\ ${ }^{1}$ Pontifícia Universidade Católica do Paraná - PUCPR - Curitiba - Brasil \\ roberto.bonanomi@totvs.com.br \\ ${ }^{2}$ Pontifícia Universidade Católica do Paraná - PUCPR - Curitiba - Brasil \\ wesley.vieira@pucpr.br \\ ${ }^{3}$ Pontifícia Universidade Católica do Paraná - PUCPR - Curitiba - Brasil \\ del.corso@pucpr.br \\ ${ }^{4}$ Pontifícia Universidade Católica do Paraná - PUCPR - Curitiba - Brasil \\ luiz.duclos@pucpr.br
}

\begin{abstract}
Resumo
Este artigo propõe uma forma alternativa para priorização dos riscos em projetos de desenvolvimento de software produto. Uma das áreas prioritárias do gerenciamento de projetos é a área de gerenciamento de riscos. A forma mais rápida para fazer uma análise qualitativa conforme Pritchard, PMI, Wideman, Schuyler e Chapman;Ward é a multiplicação da probabilidade pelo impacto dos eventos de riscos. O resultado é uma lista ordenada, onde os maiores números representam os maiores riscos. Atualmente a indústria automobilística usa o FMEA para analizar seus projetos possibilitando o levantamento, análise e a priorização das causas de falhas. Outro método analítico de avaliar fatores incerteza é a teoria Grey, que analisa o grau de relação entre as variáveis e um padrão definido como ótimo. Este trabalho é um estudo de caso em uma organização que desenvolve software produto. Os riscos foram analisados levando em conta projetos já executados e a seqüência dos eventos. Foram usados o método tradicional da probabilidade versus impacto, o método FMEA e o grau de relação da teoria Grey. O resultado demonstrou que o cálculo da priorização de riscos usando probabilidade versus impacto obteve $21 \%$ de acerto, o FMEA obteve 25\% de acerto e com a teoria Grey obteve 39\% de acerto. Outros estudos são necessários objetivando comparações entre os métodos.
\end{abstract}

Palavras-chave: gerenciamento de riscos; FMEA; teoria Grey.

\section{Introdução}

O mercado de software está crescendo no Brasil. Conforme ABES (Associação Brasileira 
das Empresas de Software), o mercado software foi avaliado como sendo o $12^{\circ}$ colocado no mundo (ABES, 2008), totalizando R\$ 15 bilhões em produtos e serviços. Desta forma, o mercado de software torna-se muito dinâmico dado que as taxas de abertura, fechamento e fusões são elevadas, pois todos os setores demandam produtos e serviços da indústria de software, conforme comprova a pesquisa da Price Waterhouse Coopers (abril/2009). Desta forma, estas organizações precisam inovar.

Segundo Porter (1999), o modo de operação de empresas bem-sucedidas é fundamentalmente idêntico, sendo que a vantagem competitiva atingida por meio de iniciativas de inovação, tais como novos desenhos de produtos, novos processos de produção, novas abordagens de marketing ou novas técnicas de gestão de pessoas. Isso remete a necessidade de se elaborar estratégias que direcionem as organizações neste tumultuado caminho para o sucesso.

De acordo com Prahalad (1997), o ponto central de uma estratégia é o de desenvolver uma série de competências essenciais e, a partir daí, criar novos produtos e serviços. O processo requer uma nova maneira de pensar, reaprender e readaptar por parte dos dirigentes da organização. Ao se criar novos produtos e serviços, pode-se dizer que são ações da organização para atender as suas estratégias. Estas ações geram projetos que precisam ser gerenciados para que se obtenha sucesso.

As organizações, conforme pesquisa do PMI (Project Management International) do Brasil em seu estudo de benchmaking em gerenciamento de projetos de 2007, apontam como uma de suas grandes dificuldades a implementação adequada na área de gerenciamento de riscos em seus projetos. Esta pesquisa aponta que no setor de software brasileiro $8 \%$ não gerenciam riscos em seus projetos, enquanto mais da metade, $60 \%$, realizam informalmente ou conforme interesse do gerente de projetos e os outros $32 \%$ são baseados em uma metodologia formal.

Os riscos são identificados e analisados, buscando-se nesta análise, validar seu poder devastador para os objetivos do projeto. Mas, dentre os mais diversos tipos de riscos e da tentativa de identificar quais merecem mais ou menos atenção ficando a dúvida se as organizações estão classificando adequadamente os riscos (ou eventos) certos. Esta priorização dos riscos está diretamente ligada à assertividade em investir tempo e dinheiro nos riscos certos e assim retornando este investimento em sucesso para os projetos.

Pode-se dizer que uma das áreas mais críticas e menos entendidas pelas organizações no gerenciamento do projeto é o gerenciamento de riscos, por ser um processo que, muitas vezes, torna-se subjetivo por ter que lidar com a probabilidade e impacto devendo ser oriundos da sua base de conhecimento organizacional e de análises comparativas internas e externas da organização.

\section{Referencial Teórico-Empírico}


Gerenciar projetos é uma prática muito antiga. Conforme Vicentinho (1997) os projetos vêm sendo feitos há mais de 2700 A.C., pois as construções das pirâmides do Egito antigo era um empreendimento único e empregavam milhares de pessoas em suas realizações. Estes projetos geraram um empreendimento único e demoraram até 20 anos para sua construção.

$\mathrm{O}$ gerenciamento de projetos, enquanto disciplina mais formal, tem sua origem relacionada ao setor militar no período pós-segunda guerra (CRAWFORD, 2002). A partir de então, tem sido cada vez mais utilizado, estando sua implantação em evidência a partir de meados da década de 1990. Vale ressaltar que uma das grandes vantagens do gerenciamento de projetos é que ele não é restrito a projetos gigantescos. Ele pode ser empregado em projetos de qualquer complexidade, orçamento e tamanho em qualquer linha de negócios (VARGAS, 2003).

As técnicas de gerenciamento de projeto, rapidamente espalharam-se para todos os tipos de indústria. Logo, líderes de projeto procuraram novas estratégias e ferramentas para gerenciar seu crescimento e o dinamismo das mudanças em um mundo competitivo. As teorias gerais do sistema da ciência então começaram a serem aplicadas às interações do negócio (KERZNER, 2002).

$\mathrm{Na}$ visão de Kerzner (2005), projeto é um empreendimento com objetivo claramente identificável, consumindo recursos e operando sob pressões de prazo, custo e qualidade. Para o PMI (Project Management Institute) em sua publicação do PMBOK - Project Management Body of Knowledge (2008) define projeto como (...) um esforço temporário empreendido para criar um produto, serviço ou resultado exclusivo. Este caráter temporário indica que cada projeto possui um inicio e um fim bem definidos.

A indústria de software, valendo-se deste conhecimento, vem aplicando o conhecimento gerado em gerenciamento de projeto para produzir e entregar software dentro das pressões de mercado como prazo, custo e qualidade.

Autores como Kerzner (2005), Heldman (2006), Cleland e Ireland (2002), Dinsmore (2003) e o PMBOK (2008) dividem o gerenciamento de projetos em áreas de conhecimento, tais como, escopo, tempo, custo, recursos humanos, qualidade, riscos, comunicação e aquisições. As empresas que usam estas áreas de conhecimento concluem os projetos com mais assertividade, conforme pesquisa da The Standish Group (2004).

$\mathrm{Na}$ área do conhecimento de gestão de projetos este trabalho analisa a gestão dos riscos de projetos de desenvolvimento de software produto. É importante salientar que a análise é focada no desenvolvimento como parte do ciclo de vida do software produto conforme definido por Duclós (1982) e referendado por Bohem (2002). Na área do conhecimento de gestão de riscos este trabalho prioriza uma lista de riscos de forma que a empresa aplique seus recursos minimizando os riscos mais danosos ao projeto. 
Kerzner (2005) define risco como a medida da probabilidade e conseqüência de não se alcançar uma meta definida do projeto. Destaca-se que o risco é constituído da falta de conhecimento dos eventos futuros, envolvendo a noção de incerteza. Tipicamente, eventos futuros favoráveis são chamados de oportunidades e eventos desfavoráveis são chamados de riscos ou ameaças.

O gerenciamento de riscos do projeto inclui o procedimento que tratam da realização de identificação, análise, respostas, monitoramento e planejamento do gerenciamento de riscos em um projeto. A maioria desses procedimentos é atualizada durante todo o projeto.

Os objetivos do gerenciamento de riscos do projeto são aumentar a probabilidade e o impacto dos eventos positivos e diminuir a probabilidade e o impacto dos eventos adversos ao projeto. Planejar para evitar ou minimizar eventos que possam ser danosos ao projeto, antecipar-se ao que pode dar errado e procurar eliminar suas causas, e não apenas remediar supostas inevitabilidades é uma postura bastante oportuna em termos de foco estratégico no gerenciamento de projetos.

Segundo Pritchard (2005), PMBOK (2008), Wideman (1992), Chapman e Ward (1997) e Schuyler (2001) a análise de riscos possui três fatores principais:

a) a probabilidade de ocorrência de um evento;

b) o impacto da ocorrência;

c) julgamento subjetivo baseado nos dois primeiros fatores.

Os benefícios da gerência de risco são claros, segundo Boehm (1991), afirma que a gerência de risco é importante principalmente porque ajuda as pessoas a evitar desastres, evitar retrabalho, evitar cancelamento de projetos e estimular uma situação de sucesso nos projetos de software produto.

\subsection{Probabilidade versus impacto de eventos de risco}

Conforme PMBOK (2008) a análise dos riscos pode ser subdividida em duas fases, qualitativa e quantitativa. Na análise qualitativa é feito uma avaliação subjetiva da lista de riscos, podendo, conforme cada organização ser uma matriz conforme representada pela Figura 1, escala numérica, ou usando letras, já que é uma análise subjetiva conforme apresentado na Figura 2 na forma de escala relativa.

A Figura 1 demonstra um exemplo de matriz de probabilidade versus impacto e considera uma escala para impacto de $0,05,0,10,0,20,0,40$ e 0,80 e para probabilidade de 0,1 a 0,9 . 
Figura 1 - Exemplo de Matriz versus Probabilidade (escala numérica)

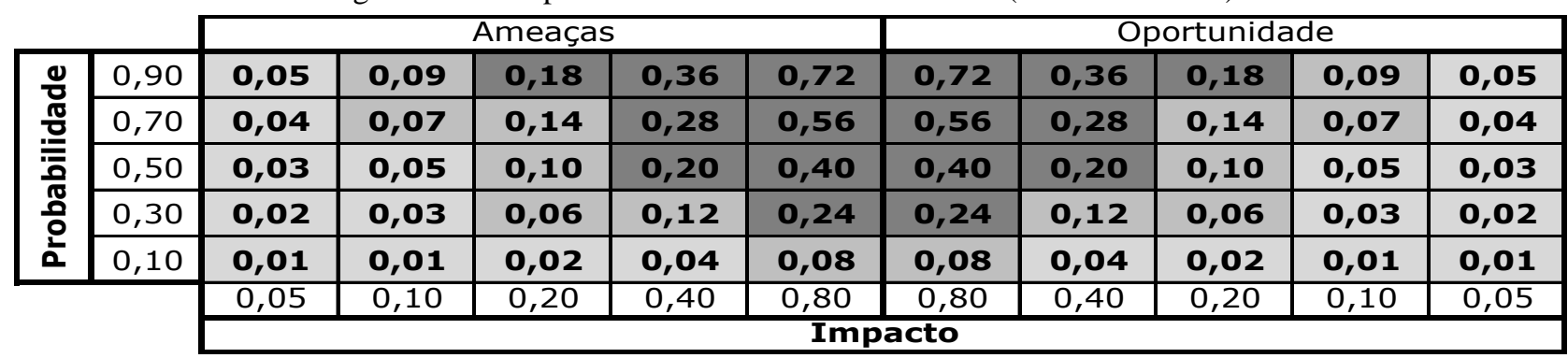

Fonte: Adaptado PMBOK (2008)

Na Figura 1 o uso da equação (1) definida a seguir, determina os valores da matriz, cada organização pode escolher os valores de impacto que melhor representam a escala. Para os valores da probabilidade, estes devem estar entre 0 e 1.

Já na Figura 2, podem-se usar letras para representar o fator de probabilidade e o impacto de forma relativa. A letra $\mathrm{B}$ seria um fator de probabilidade ou impacto baixo, a letra $\mathrm{M}$ um fator $\underline{\text { médio e a letra A um fator alto. }}$

Figura 2 - Exemplo de Matriz versus Probabilidade (escala relativa)

\begin{tabular}{|c|c|c|c|c|}
\hline \multirow{3}{*}{ 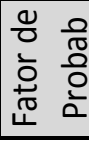 } & A & $A B$ & AM & AA \\
\hline & $\mathbf{M}$ & $\mathrm{MB}$ & $\mathrm{MM}$ & $\mathrm{MA}$ \\
\hline & B & BB & BM & $\mathrm{BA}$ \\
\hline & & B & $\mathbf{M}$ & A \\
\hline
\end{tabular}

Fonte: Dinsmore e Cavalieri (2003)

Nos dois exemplos descritos anteriormente, referentes a matriz de impacto versus probabilidade, vê-se que há a geração da exposição a risco que pode ser calculada usando-se a equação (1).

$$
E=P x I
$$

Onde:

$E$ : exposição aos riscos;

$P$ : probabilidade (ou fator de probabilidade) da ocorrência;

I: impacto nos objetivos do projeto.

A probabilidade multiplicada pelo impacto (valor da perda) dá-se o nome de exposição ao risco conforme designado na equação (1). Em projetos, um evento ou ação que é considerado um risco, deve-se ter uma perda associada, uma chance ou alguma escolha (CHARETTE, 1990), ou seja, o risco pode ser modificado por uma ação pensada e planejada. Para o efetivo gerenciamento dos riscos é associado algumas informações como descrição, um responsável, planos de ação e o 
gatilho do risco, ou seja, como pode perceber que o risco está próximo ou prestes a acontecer.

Colocando-se os riscos em ordem decrescente de sua exposição, pode-se obter uma lista ordenada para o qual a organização deve focar sua atenção em qualificá-los, ou seja, tornando estas matrizes valores financeiros de impacto no projeto. Contudo, deve-se avaliar qual o esforço para tornar esta exposição um valor financeiro para o projeto.

O PMBOK (2008) recomenda que sejam usados alguns riscos classificados com alta exposição para então fazer uma análise quantitativa. Esta análise deve usar diversas técnicas de cálculo, tais como simulação de Monte Carlo, árvore de decisão e valor monetário esperado.

\subsection{FMEA - Análise dos modos de falha e efeitos}

Avaliando as formas de se classificar riscos, a literatura e a indústria, apresenta uma técnica chamada FMEA - Failure Mode and Effect Analysis. Esta técnica surgiu em 1949 para avaliar equipamentos no exercito americano e segundo Puente et al. (2001), no início da década de 60, durante o projeto Apollo, a NASA aprimorou a técnica inicial com método para identificar e classificar, de forma sistemática, as falhas potenciais em sistemas (produto ou processos) antes que as mesmas ocorressem. Mais tarde, em 1972 a Ford Motor Company introduziu o FMEA de processo na indústria automobilística e difundiu seu uso para toda rede de fornecedores com a norma QS 101.

Atualmente, o FMEA é usado nas mais diversas atividades, desde indústria química, biológica, ambiental, alimentícia, etc. Em seu trabalho, Vandebrande (1998), demonstra que com algumas adaptações pode-se usar esta ferramenta de avaliação de riscos nas mais diversas áreas. A lógica aplicada por Vandebrande (1998), será usada aqui, para prevenção de riscos em projetos de desenvolvimento de software produto, prevenindo problemas com todas as fases de desenvolvimento do software produto.

Os benefícios do desenvolvimento e manutenção dos FMEAs eficazes são elencados por Palady (2004), entre eles a economia nos custos e tempo de desenvolvimento serve como guia para o planejamento de testes mais eficientes. Também fornece uma rápida referência para a resolução de problemas, reduz mudanças na engenharia, aumenta a satisfação do cliente, captura e mantém o conhecimento do produto e do processo na organização, reduz eventos não previstos durante o planejamento de um processo, identifica as preocupações de segurança a serem abordadas entre outros.

O método FMEA traduz, em uma seqüência lógica e sistemática, a avaliação das formas possíveis pela qual um sistema ou processo está mais sujeito a falhas. O FMEA considera as variáveis, severidade $(\mathrm{S})$ das falhas, a frequiência como as mesmas ocorrerem $(\mathrm{O})$ e, como 
eventualmente poderiam ser detectadas (D), antes de chegarem às reclamações dos clientes. Assim, com base nestes três índices: severidade, ocorrência e detecção, o método leva a uma priorização de quais modos de falha do produto podem causar maior risco aos clientes e à própria organização.

As variáveis acima citadas podem ser classificadas em tabelas conforme cada indústria ou projeto onde serão aplicadas. As Figuras de 3 a 5, respectivamente, demonstram estas classificação conforme padrão usado pela Ford Motor Company. A Severidade de um evento está demonstrada no Quadro 1.

\begin{tabular}{|l|l|c|}
\hline Severidade & \multicolumn{1}{|c|}{ Cuadro 1 - Critério de Análise para Severidade das Falhas } & Índice de Falha \\
\hline Perigoso & $\begin{array}{l}\text { É muito perigosa, risco a vida ou não conformidade com } \\
\text { legislação. Sem aviso prévio }\end{array}$ & 10 \\
\hline Sério & $\begin{array}{l}\text { É muito perigosa, risco a vida ou não complacente com } \\
\text { legislação. Com aviso prévio }\end{array}$ & 9 \\
\hline Importante & Produto inoperável, com perdas das funções básicas & 8 \\
\hline Impactante & Desempenho do produto sofre impacto, podendo não operar & 6 \\
\hline Significativo & $\begin{array}{l}\text { Desempenho do produto é degradado. Funções podem não } \\
\text { operar. }\end{array}$ & 5 \\
\hline Moderado & Moderado efeito sobre o produto, requerendo reparos & 3 \\
\hline Baixo & $\begin{array}{l}\text { Pequeno defeito no desempenho do produto, não necessitando } \\
\text { de reparos }\end{array}$ & 2 \\
\hline Insignificante & Efeito insignificante no produto, não necessitando de reparos & 1 \\
\hline Desprezível & $\begin{array}{l}\text { Efeito insignificante no produto, não necessitando de reparos, } \\
\text { notado pelo cliente }\end{array}$ & \\
\hline $\begin{array}{l}\text { Muito } \\
\text { desprezível }\end{array}$ & Efeito desprezível no produto, não necessitando de reparos & 1 \\
\hline
\end{tabular}

Fonte: Adaptado da FORD MOTOR COMPANY (1988)

Este Quadro 1 representa a classificação da severidade de um risco onde número 1 é considerado muito baixo e o número 10 altamente perigo conforme critério. O Quadro 2 demonstrada as classificações para ocorrência da falha ou risco.

Quadro 2 - Critério de Análise para Índice de Ocorrência das Falhas

\begin{tabular}{|l|c|c|}
\hline Ocorrência de falha & Taxas possíveis de falha & Índice de Ocorrência \\
\hline \multirow{3}{*}{ Extremamente alta } & $\geq 1 \mathrm{em} 2$ & 10 \\
\hline \multirow{3}{*}{ Moderada } & $1 \mathrm{em} 3$ & 9 \\
\cline { 2 - 3 } & $1 \mathrm{em} 8$ & 8 \\
\cline { 2 - 3 } & $1 \mathrm{em} 20$ & 7 \\
\hline \multirow{3}{*}{ Baixa } & $1 \mathrm{em} 80$ & 6 \\
\cline { 2 - 3 } & $1 \mathrm{em} 400$ & 4 \\
\hline Quase impossível & $1 \mathrm{em} 2.000$ & 3 \\
\cline { 2 - 3 } & $1 \mathrm{em} 15.000$ & 2 \\
\cline { 2 - 3 } & $1 \mathrm{em} 150.000$ & 1 \\
\hline
\end{tabular}

Fonte: Adaptado de FORD MOTOR COMPANY (1988) 
No Quadro 2, percebe-se que a classificação da ocorrência de um risco pode ser representada por taxa de falhas onde 1 é considerado quase impossível e 10 extremamente provável que ocorra. Os critérios de classificação para Detecção das falhas estão listados no Quadro 3 mostrando os índices de falhas que varia de 1 até 10 .

Quadro 3 - Critério de Análise para Índice de Detecção das Falhas

\begin{tabular}{|l|l|c|}
\hline Detecção & \multicolumn{1}{|c|}{ Possibilidade de detecção da falhas } & Índice de Falha \\
\hline Totalmente incerta & Quase impossível detectar a falha & 10 \\
\hline Muito remota & Chance muito remota de detectar a falha & 9 \\
\hline Remota & Chance remota de detectar a falha & 8 \\
\hline Muito baixa & Chance muito baixa de detectar a falha & 7 \\
\hline Baixa & Chance baixa de detectar a falha & 6 \\
\hline Moderada & Há moderada chance de detectar a falha & 5 \\
\hline Moderadamente alta & Moderadamente alta de se detectar a falha & 4 \\
\hline Alta & Alta chance de se detectar a falha & 3 \\
\hline Muito alta & Há chance muito alta de se detectar a falha & 2 \\
\hline Quase certa & É quase certo que a falha será detectada & 1 \\
\hline
\end{tabular}

Fonte: Adaptado da FORD MOTOR COMPANY (1988).

Assim, o Quadro 3 evidencia a classificação sobre a possibilidade de detectar uma falha ou risco, variando de 1 quase certa (facilmente detectada) até 10 que será considerado quase impossível de ser detectada (difícil).

Um risco é considerado alto quando tiver um impacto significativo no produto ou no cliente, juntamente com uma ocorrência provável e uma baixa capacidade de ser detectado antes de seu efeito ser percebido pelo cliente. O procedimento mais utilizado para se medir o risco associado à cada modo de falha é a multiplicação da pontuação obtida para as classificações da severidade (S), ocorrência (O) e detecção (D). Com isso, tem-se uma escala que vai de 1 até 1000 pontos, sendo número 1 para baixíssimo risco e o número 1000 um risco altamente crítico. Esta pontuação é chamada de número (ou grau) da prioridade do risco (NPR) ou do inglês Risk Priority Number $(R P N)$. Será adotada a sigla original $R P N$ que pode ser mensurado por meio da expressão (2).

$$
R P N=0 \times \mathrm{S} \times \mathrm{D}
$$

Onde:

RPN: Risk Priority Number,

O: ocorrência;

S: severidade;

D: detecção. 
Um ponto importante a ser considerado depois da construção do FMEA é a fase de acompanhamento. Para fazer o acompanhamento, outras ferramentas de suporte à qualidade e confiabilidade devem ser usadas. Geralmente os dados devem ser analisados utilizando-se de métodos estatísticos. Se não houver a capacidade de utilizar as ferramentas de suporte e o compromisso com o acompanhamento dentro da equipe, pouco ou nenhum benefício pode ser esperado do FMEA, restam somente formulários para simples auditoria por parte da indústria.

Há uma série de autores que criticam a forma com que é calculado o $R P N$, tais como Franceschini e Galetto (2001), Gilchrist (1993), Bowles (1998) e Chin et al. (2007). Para minimizar estes impactos, autores ou instituições como Gilchrist (1993), Bem-Daya e Raouf (1996), KEMA (1996), Puente (2001), Chang et al. (2001) propuseram novas formas de cálculo do $R P N$, que não serão apresentadas neste estudo.

\subsection{A teoria Grey}

Proposta por Deng (1989), a teoria Grey, ou análise de relação Grey, é uma técnica eficaz, que pode ser usada para auxiliar a tomada de decisões em um ambiente de incertezas e em situações com vários atributos por meio do exame de similaridade de cada alternativa com uma solução ideal. Neste trabalho, os atributos serão ocorrência, severidade e detecção os fornecidos pelo FMEA.

A teoria Grey permite uma mensuração para analisar a relação entre séries qualitativas e quantitativas discretas. A abordagem é baseada pelo nível de similaridade e variabilidade entre todos os fatores para estabelecer sua relação. A análise relacional sugere como fazer previsões e tomar decisões. Exemplos de uso deste modelo podem ser encontrados na agricultura, economia, hidrologia, meteorologia e gerenciamento de maneira geral. Ver trabalhos de Leephakpreeda (2008), Wang e Hsu (2008), Wei e Chung (2003) e também o trabalho de HE et al. (2008). Para WU (2002), a teoria relacional Grey tradicional inclui seguir seis passos:

a) construir uma matriz $(X)$ de decisão inicial, conforme definida em (3). Assumindo que são $n$ sequiências e são caracterizadas por $m$ critérios, ou fatores. Assim, cada fator de decisão forma um vetor da matriz $\left\{x_{1}(1), x_{1}(2), \ldots x_{1}(m)\right\}$ que são as séries, neste trabalho $x_{1}$ representa os eventos de riscos ou falhas.

$$
X=\left[\begin{array}{c}
x_{1} \\
x_{\mathbf{2}} \\
\ldots \\
\ldots \\
x_{n}
\end{array}\right]=\left[\begin{array}{cccc}
x_{1}(1) & x_{1}(2) & \ldots & x_{\mathbf{1}}(m) \\
x_{\mathbf{2}}(1) & x_{\mathbf{2}}(2) & \ldots & x_{\mathbf{2}}(\mathrm{m}) \\
\ldots & \ldots & \ldots & \ldots \\
\ldots & \ldots & \ldots & \ldots \\
x_{n}(1) & x_{n}(2) & \ldots & x_{n}(m)
\end{array}\right]
$$

Onde $x_{i}(j)$, é uma entidade na $i$ sequência de dados e que correspondem ao critério $j$; 
b) normalização dos fatores bases, e são caracterizados por 3 tipos de critérios: 1) fator maior é melhor, representado por $f 1$ na equação (4); 2) fator menor é melhor, representado por $f 2$ conforme equação (5) e nominal é o melhor fator $f 3$ apresentado na equação (6). Neste trabalho será usada a normalização $f 2$, visto que quanto menor o fator melhor, pois os riscos podem ser considerados mais baixos.

$$
\begin{gathered}
f 1=x_{i}^{\prime}(j)=\frac{x_{1}(j)-\min _{i=1}^{n}\left[x_{i}(j)\right]}{\max _{i=1}^{n}\left[x_{i}(j)\right]-\min _{i=1}^{n}\left[x_{i}(j)\right]} \\
f 2=x_{i}^{\prime}(j)=\frac{\max _{i=1}^{n}\left[x_{i}(j)\right]-x_{i}(j)}{\max _{i=1}^{n}\left[x_{i}(j)\right]-\min _{i=1}^{n}\left[x_{i}(j)\right]} \\
f 3=x_{i}^{\prime}(j)=1-\frac{\left[x_{i}(j)-x_{o b j}(j)\right]}{\max \left[\max _{i=1}^{n}\left[x_{i}(j)\right]-x_{o b j}(j)_{s}-x_{o b j}(j)-\min _{i=1}^{n}\left[x_{i}(j)\right]\right\}}
\end{gathered}
$$

Onde $x_{o b j}(j)$ é o valor meta para o fator de $j$ deve estar de acordo com a expressão (7).

$$
\operatorname{mix}_{i=1}^{n}\left[x_{i}(j)\right] \leq x_{o b j}(j) \leq \max _{i=1}^{n}\left[x_{i}(j)\right]
$$

Com o uso da normalização, todos os três tipos de critérios foram transformados em o melhor tipo para o problema a ser resolvido, onde o valor máximo é 1 . No caso do uso com o FMEA deve-se usar a equação (5), pois para minimizar os riscos, quanto menor o valor dos fatores melhor. Forma-se assim, a série padrão $x_{0}=\left\{x_{0}(1), x_{0}(2), \ldots x_{0}(m)\right\}=\{1,1, \ldots 1\}$;

c) neste passo, deve-se construir a matriz normalizada $x^{g}$ de acordo com o critério selecionado no passo anterior, usando uma das equações, (4), (5) ou (6), respectivamente, tem-se as equações (8) e (9):

$$
\begin{aligned}
& x^{y}=\left[\begin{array}{cccc}
x_{1}^{y}(1) & x_{1}^{y}(2) & \ldots & x_{1}^{y}(m) \\
x_{2}^{y}(1) & x_{2}^{y}(2) & \ldots & x_{2}^{y}(m) \\
\ldots & \ldots & \ldots & \ldots \\
\ldots & \ldots & \ldots & \ldots \\
x_{m}^{y}(1) & x_{n}^{y}(2) & \ldots & x_{m}^{y}(m)
\end{array}\right] \\
& x_{0}^{\prime}=x_{0}^{\prime}(1), x_{0}^{\prime}(2), \ldots x_{0}^{y}(m)
\end{aligned}
$$


Onde $x_{0}^{\prime}(0)$ é o valor referência na relação para o fator $j$ e foi determinada pela melhor normalização de cada fator. Isto é, $x_{n}^{x}(j)=\max _{i=1}^{n}\left[x_{i}(j)\right]$. Para esta pesquisa, são os menores valores de ocorrência, severidade e detecção;

d) calcular a diferença entre a entidade normalizada e o valor de referência, construindo, assim, uma matriz de diferença, conforme evidenciado nas equações (10) e (11), respectivamente:

$$
\begin{aligned}
& \Delta_{0 n}(j)=\left[x_{0}^{n}(j)-x_{i}^{\prime}(j)\right] \\
& \Delta=\left[\begin{array}{cccc}
\Delta_{01}(1) & \Delta_{01}(2) & \ldots & \Delta_{01}(m) \\
\Delta_{02}(1) & \Delta_{02}(2) & \ldots & \Delta_{02}(m) \\
\ldots & \ldots & \ldots & \ldots \\
\ldots & \ldots & \ldots & \ldots \\
\Delta_{0 n}(1) & \Delta_{0 n}(2) & \ldots & \Delta_{0 n}(m)
\end{array}\right]
\end{aligned}
$$

e) calcular o coeficiente relacional Grey para cada um dos itens dos fatores conforme equação (12):

$$
\gamma_{\circ i}(j)=\frac{\Delta_{i}(\min )+\rho \times \Delta_{i}(\max )}{\Delta_{0 i}(j)+\rho \times \Delta_{i}(\max )}
$$

Onde:

$$
\begin{aligned}
& \Delta_{i}(\min )=\min _{i=1}^{n} \min _{j=1}^{m} \Delta_{0 i}(j) \\
& \Delta_{i}(\max )=\max _{i=1}^{n} \max _{j=1}^{m} \Delta_{0 i}(j)
\end{aligned}
$$

$\rho(0 \leq \rho \leq 1)$ é conhecido como coeficiente de distinção. Quando menor $\rho$ mais distinção ele possui. Na maioria das situações é usado valor 0,5 por ter efeito moderado e boa estabilidade para equação conforme Chang e Lin (1999);

f) calcular o grau relacional Grey para cada um dos itens dos fatores da série conforme equação (12). Antes de calcular, é necessário definir o critério de peso para cada um dos fatores $\begin{aligned}\{\alpha(1), \alpha(2), \ldots \alpha(m)\}, \text { onde a } & \sum^{m} \alpha=1 \\ \tau_{0 i} & =\sum_{j=1}^{m}\left[\alpha(j) \times \gamma_{o i}(j)\right]\end{aligned}$

O critério de peso $\alpha$ deve ser definido em cada contexto aplicação. 
Grau relacional de Grey ( $\left.\tau_{0 i}\right)$ indica a magnitude da correlação, ou similaridade, medida entre a comparação das seqüências (ou seja, cada fator da série), e a sequência de referência equação (9). Portanto, o grau relacional de Grey calculado, das séries definidas a matriz $X$ da equação (10), podem ser priorizadas aquelas com maior grau de relação, pois representam a melhor solução por estar mais próximo da série ideal.

No caso desta pesquisa, no qual pretende-se priorizar as falhas mais prejudiciais ao protfolio de projetos, quando menor o grau de correlação, mais distante ele está da sequência da série ideal, sinalizando que se deve atuar primeiro nestes eventos potenciais.

\section{Metodologia da pesquisa}

Esta pesquisa visa realizar um levantamento dos principais riscos envolvidos em projetos de desenvolvimento de software produto na Empresa Alpha (proxy para empresa analisada) objetivando a proposição de uma forma diferenciada para classificar os riscos destes projetos.

Assim, como na Empresa Alpha, a maioria das organizações desenvolvedoras de software produto, 92\%, conforme pesquisa PMI (2007), que gerenciam riscos utiliza-se de uma forma clássica para se avaliar riscos atualmente, que leva em consideração a probabilidade de um evento ocorrer multiplicado pelo seu impacto, conforme visto na equação (1). Esta multiplicação gera o fator de exposição ao risco e por conseqüência a lista de risco a ser priorizada.

Para realização deste trabalho pode-se observar no Quadro 4, de forma resumida a metodologia usada.

Quadro 4 - Metodologia utilizada neste trabalho

\begin{tabular}{|c|c|c|}
\hline Suporte Instrumental & \multicolumn{2}{|c|}{ - $\quad$ Planilha eletrônica Excel } \\
\hline $\begin{array}{c}\text { Suporte } \\
\text { Metodológico da } \\
\text { Pesquisa }\end{array}$ & $\begin{array}{l}\text { Classificação } \\
\text { da pesquisa }\end{array}$ & $\begin{array}{l}\text { Abordagem: Quantitativa } \\
\text { Quanto à Natureza: Pesquisa Aplicada } \\
\text { Quanto aos Objetivos: Explicativa } \\
\text { Quanto aos Procedimentos: Estudo de caso } \\
\text { Quanto aos Meios: Pesquisa ex-post facto } \\
\text { Quanto ao Aspecto Temporal: Corte Transversal } \\
\text { Quanto aos Meios de Investigação: Pesquisa Documental e Pesquisa } \\
\text { Bibliográfica } \\
\text { Quanto ao Método de Amostragem: Não Probabilístico ou Não Aleatório }\end{array}$ \\
\hline & $\begin{array}{l}\text { Campo de } \\
\text { aplicação }\end{array}$ & $\begin{array}{l}\text { Gerenciamento de Riscos em projetos de desenvolvimento de software da } \\
\text { organização ALPHA. }\end{array}$ \\
\hline
\end{tabular}

No Quadro 4, conforme destaca Yin (2005), os estudos de caso representam a estratégia preferida quando se colocam questões do tipo "como" e "por que", quando o pesquisador tem pouco controle sobre os acontecimentos e quando o foco se encontra em fenômenos contemporâneos inseridos em algum contexto da vida real. Os estudos de caso podem ainda serem utilizados em pesquisas exploratórias, quanto descritivas ou explicativas. 
Quanto aos meios de investigação refere-se a uma pesquisa de análise documental, visto que serão avaliadas as informações arquivadas dos projetos finalizados ou em andamento no período supracitado. Também pode-se caracterizar esta pesquisa como sendo uma pesquisa bibliográfica, dado que será realizado um levantamento bibliográfico sobre as principais métricas de riscos existentes para gerenciamento de projetos para poder fazer a comparação com as encontradas nos projetos a serem analisados.

Quanto ao método de amostragem, esta pesquisa utiliza-se do processo de amostragem não probabilístico ou não aleatória, visto que serão selecionados todos os projetos de desenvolvimento de software da empresa Alpha de forma intencional no período previamente considerado.

A amostra coletada caracteriza-se como não probabilística por conveniência, em função da proximidade do autor da pesquisa com a organização pesquisada, bem como, em razão do seu conhecimento a respeito das técnicas de gerenciamento de projetos.

A empresa Alpha possui ferramenta de GED - Gerenciamento Eletrônico de Dados, que é um software comercial da própria empresa, onde são armazenados todos os tipos de documentos. Os documentos de gerenciamento dos projetos de desenvolvimento de software produto também são armazenados nesta ferramenta.

Com base na avaliação da documentação existente, são identificados os riscos que estão definidos e detalhados nas planilhas de acompanhamento de riscos. Adicionalmente, todos os outros documentos do projeto são fontes de informação de problemas enfrentados (eventos de riscos não identificados) e avaliar quais foram as ações tomadas.

Em uma planilha eletrônica, serão levantados todos os riscos existentes nos projetos selecionados. Com base na matriz de probabilidade versus impacto citada no referencial teórico será feito o cálculo de exposição a riscos de cada evento e a devida ordenação dos riscos por ordem de importância (do maior para o menor). Estes mesmos riscos serão avaliados conforme definição da técnica FMEA, onde será calculado o $R P N$ para cada risco e da mesma forma ordenados do maior para o menor risco. Ainda com o uso de planilha eletrônica para seguir os passos indicados por WU (2002). Ao final, pretende-se comparar a ordenação das três formas de priorizar riscos com a ordem em que os riscos tornaram-se um fato, sendo necessárias ações para de alguma forma eliminar o problema ou diminuir o impacto gerado.

\section{Apresentação e análise dos resultados}

A empresa Alpha foi fundada na década de 70 para atender as necessidades de software da região de Joinville. Logo se tornando líder de mercado na região. Os anos 80 representaram a expansão para sul e sudeste do país. Com parcerias de fornecedores de ferramentas de 
desenvolvimento, logo se instalou por todo pais. Ao final dos anos 90 já possuía clientes por todo o continente americano, chegando a um faturamento em $\mathrm{R} \$ 100$ milhões.

O levantamento dos dados de projetos da empresa Alpha contou com ferramenta de GED Gerenciamento Eletrônico de Dados, que se trata de um software comercial da própria organização. Nesta ferramenta são armazenados os mais variados tipos de documentos. Assim, os documentos de gerenciamento dos projetos de desenvolvimento de software produto também estão disponíveis nesta ferramenta, facilitando a comunicação e o uso das informações.

A organização possuía em seu portfolio de projetos de desenvolvimento no momento da pesquisa 261 projetos. Os dados foram levantados desde o ano de 2002, com isso, pode-se sugerir que há uma boa experiência em gerenciamento de projetos na organização. Isso não quer dizer que antes desta data não se gerenciava projetos, mas sim que existiam outras formas de armazenamento da informação de projetos. Para efeitos dessa pesquisa, faz-se um corte em termos temporais.

Como características básicas para esta pesquisa, os projetos selecionados possuem os seguintes fatores:

a) possuir mais de 2.000 horas em desenvolvimento de software produto;

b) ter como base desenvolvimento em alguma tecnologia nova, neste caso, serão usadas projetos com inovação tecnológica na interface com usuário e voltados para WEB (uso via internet). Atualmente, este é o grande marco de inovação para produtos tipo commodities como são os ERP Enterprise Resource Planning.

Neste sentido, foram selecionados 27 projetos, tal como encontra-se na Tabela 1, entre os que já foram encerrados, suspensos e aqueles que estão em fases de fechamento (mais de $75 \%$ do projeto concluído). Todos estes projetos são projetos de desenvolvimento de software produto para compor o produto ERP - Enterprise Resource Planning, não sendo considerados pacotes específicos ou algum projeto de ajuste para algum cliente. Estes projetos têm o objetivo comum de tratar a interface gráfica com nova tecnologia, usando como base o ERP já pré-existente.

Tabela 1: Número de Projetos da Organização Alpha para a Pesquisa

\begin{tabular}{ccc}
\hline Situação Projeto & Número de Projetos & Percentual \\
\hline Em Desenvolvimento & 3 & $11 \%$ \\
Encerrados & 22 & $81 \%$ \\
Suspensos & 2 & $7 \%$ \\
\hline
\end{tabular}

Fonte: Autor com base nas informações disponibilizadas pela organização

Na Fundamentação Teórica é citada a necessidade de um plano de gerenciamento de riscos. Nele são definidos os valores que irão compor as probabilidades, impactos e a matriz exposição. Para probabilidade os valores serão entre 0,10 a 0,99 , visto que no caso de uma probabilidade $100 \%$ 
não é mais um evento de risco e sim um fato ou evento que já ocorreu e que precisa ser tratado. Para o Impacto, a organização usa a definição operacional abaixo:

$$
\begin{array}{ll}
\text { 9-Catastrófico } & \text { - Muito Alto } \\
7 \text { - Danoso } & - \text { Alto } \\
\text { 5-Expressivo } & - \text { Médio } \\
\text { 3-Considerável } & \text { - Baixo } \\
1 \text { - Insignificante } & \text { - Muito Baixo }
\end{array}
$$

De acordo com as especificações de riscos mencionadas anteriormente, no plano de gerenciamento de riscos é montada a matriz que representa as combinações de probabilidade e impacto (P x I) que levam à classificação dos riscos como de prioridade baixa, moderada ou alta. Conforme indicado na Figura 1, onde o gerente de projeto e a organização avaliam quais ações irão tomar para cada nível de exposição encontrada no levantamento.

Para cálculo do RPN do FMEA, conforme (2), esta pesquisa fez a seguinte equalização das variáveis entre os dois métodos de priorização de riscos. A probabilidade, citada no método tradicional, no FMEA será a Ocorrência, esta probabilidade para que seja adequada a tabela de Ocorrência citada será multiplicada por 10, para tornar este variável conforme Quadro 2.

A variável Impacto foi estimada por meio do FMEA e no caso da variável Severidade foram mantidos os valores inicialmente levantados inicialmente. Para o cálculo do RPN é necessário uma terceira variável que é a Detecção. Esta variável refere-se a análise dos dados originais levantados buscando-se na descrição do gatilho dos riscos, ou seja, o quanto é fácil ou difícil ser detectado antecipadamente, alocando em uma faixa de 1 até 10. Cada risco terá as três variáveis formando uma matriz que necessária para a etapa seguinte.

A etapa do cálculo da teoria Grey, utiliza-se a matriz gerada na etapa do FMEA, onde tem-se as variáveis de Severidade, Impacto e Detecção, gerando a matriz (3). O critério de peso $\alpha$ neste trabalho leva em conta a necessidade de se priorizar a variável severidade ou impacto. Estes pesos podem ser observados no apêndice A.

Para esta pesquisa foi criado um instrumento de pesquisa onde seriam inseridos todos os dados e seus respectivos cálculos, conforme as equações apresentadas no referencial teórico. O quadro completo com todos os 28 riscos levantamentos e que são relevantes para esta pesquisa estão descritos no Apêndice A. Com base neste instrumento de pesquisa é que são apresentados os dados resultantes da comparação. 
Observa-se que para a ordenação do GR - Grau de Relação da teoria Grey, quanto menor o número, mais o resultado estão se afastando da série padrão $\{1,1,1\}$. Representando assim um risco maior que deve ser tratado primeiro.

Os números de GR próximos de 1, são os mais próximos da série padrão não representando perigo imediato para análise. O uso de planilha eletrônica facilita o ajuste para projetos ou organizações que queiram priorizar outras variáveis como Detecção ou Ocorrência, o que não é permitido no método FMEA.

A Tabela 2 demonstra à análise comparativa dos métodos de priorização avaliados, mostra certa vantagem no acerto da priorização feita pelo método FMEA em relação a chamado método tradicional, $21 \%$ contra $25 \%$. O uso da teoria Grey, representou 39\% dos acertos na priorização dos riscos em relação ao que realmente aconteceu no portfolio. Mas, há de se destacar que em 32\% dos eventos de riscos não houve acerto por nenhum dos métodos usados. Houve acertos comuns entre os métodos.

Tabela 2: Análise Comparativa dos Métodos de Priorização Avaliados

\begin{tabular}{c|c|c}
\hline Método de Priorização & Número Acertos & $\%$ Acerto \\
\hline Probabilidade verus Impacto & 6 & $21 \%$ \\
\hline FMEA & 7 & $25 \%$ \\
\hline Grau de Relação Grey & 11 & $39 \%$ \\
\hline Não houve acerto & 9 & $32 \%$ \\
\hline
\end{tabular}

Fonte: Autor com base no cálculo usando o instrumento de pesquisa

O uso da teoria Grey para priorização de riscos, demonstrada nesta pesquisa e resumida na Tabela 2, melhora em até $18 \%$ o acerto da priorização dos riscos e relação a priorização pelo método Probabilidade versus Impacto.

\section{Considerações finais e recomendações}

A crescente preocupação com o gerenciamento de riscos tem um grande motivo, a globalização e competitividade entre organizações. Isto faz com que as pesquisas acadêmicas devam realizar estudos relevantes para aprimorar o gerenciamento de riscos. Melhorias nas ferramentas e na forma de priorizar e analisar riscos pode assegurar que o gerenciamento de riscos continuará a se desenvolver contribuindo para o sucesso dos projetos onde são efetivamente usados. Este trabalho se propôs a este fim.

O uso da teoria Grey aliada ao FMEA para fazer a priorização de riscos em projetos de desenvolvimento de software é novo e requer um uso mais intensivo. O estudo de caso apresentado foi um levantamento de dados ocorridos ex-post fact, analisando os riscos encontrados e refazendo 
os cálculos de priorização. A aplicação deste método desde o inicio do gerenciamento do projeto pode contribuir para avaliar mais efetivamente os resultados encontrados.

Este estudo de caso mostrou que, usando o FMEA aliado a teoria Grey como ferramenta de priorização de riscos, obteve-se um ganho em termos de acerto na classificação dos riscos. O percentual de acerto $18 \%$ maior em relação ao modo chamado de tradicional na priorização de riscos de projetos de software produto é um avanço. As organizações precisam estar dispostas a inovar usando formas alternativas disponíveis para melhorar a sua assertividade no gerenciamento de riscos.

Como esta pesquisa foi realizada em uma única organização não há como fazer qualquer tipo de generalização em relação aos dados obtidos. Organizações têm graus de amadurecimento diferentes em gerenciamento de projetos e mais especificamente em gerenciamento de riscos. Assim, esta pesquisa está delimitada a uma organização que tem cerca de 10 anos de experiência em gerenciamento de projetos, mas somente próximo de cinco anos em gerenciamento de riscos nos projetos.

Este estudo pode ser aplicado a projetos de outras organizações de desenvolvimento de software. Estas organizações possuem níveis de amadurecimento diferentes e, portanto, resultados precisam levar em conta tal amadurecimento. Estes estudos podem validar ou não os resultados aqui obtidos.

\begin{abstract}
This article proposes an alternative way to prioritize risks in projects of software development product. One of the priority areas in project management is the risk management area. The fastest way to make a qualitative analysis according to Pritchard, PMI, Wideman, and Schuyler Chapman, Ward is the multiplication of the probability by the impact of risk events. The result is an ordered list, where the biggest numbers represent the highest risks. Currently the automotive industry uses the FMEA to analyze their designs enabling the assessment, analysis and prioritization of the causes of failures. Another analytical method to evaluate uncertainty factors is the Grey theory, which analyzes the relationship degree between variables and an established standard. This paper is a case study in an organization that develops software product. The risks were analyzed taking into account projects already executed and the sequence of events. It was used the traditional method of probability versus impact, the FMEA method and the relationship degree of Grey theory. The result showed that prioritization risk calculation using probability versus impact had $21 \%$ of accuracy, FMEA had 25\% of accuracy and with the Grey theory had 39\% of accuracy. Further studies are necessary to refine the comparisons between methods.
\end{abstract}

Key-words: risk management; FMEA; Grey theory.

\title{
Referências
}

BEN-DAYA, M.; RAOUF, A. A revised failure mode and effects analysis model. International Journal of Quality Reliability Management v. 13, n. 1, 1993. p. 43-47.

crossef

BOWLE, J. B.; BONNELL, R. D. Failure mode, effects, and criticality analysis ( What It Is and How To Use It ). Annual Reliabiity and Maintenability Symposium, 1998. 
BOEHM, B. Software risk management: principles and practices. IEEE Software. v. 8, Jan. 1991, p. 32 - 41.

cross ${ }^{\text {ref }}$

. Software engineering economics. In: BROY, M.; DENERT, E. (Org.) Software pioneers: contributions to software engineering. Berlin: Springer, 2002.

CHANG, C.; LIU, P.; WEI, C. Failure mode and effects analysis using Grey theory. Integrated Manufacturing Systems. v.12, n.3, 2001, p. 211-216.

cross ref

CHAPMAN, C.; WARD, S. Project risk management: processes, techniques and insights. New York: John Wiley \& Sons, 1997.

CHARETTE, R. Application strategies for risk analysis. New York: MultiScience Press. 1990, p. 17-21.

CLELAND, D. I.; IRELAND, L. R. Gerência de projetos. Rio de Janeiro: Reichmann \& Affonso, 2002.

CRAWFORD, J. K. The strategic project office: A Guide to Improving Organizational Performance. New York: Marcel Dekker Inc, 2002.

DINSMORE, P.; CAVALIERI A. Como se tornar um profissional em Gerenciamento de Projeto: livro-base de preparação para certificação PMP. Rio de Janeiro: QualityMark. 2003.

Estudo de benchmarking em gerenciamento de projetos Brasil - 2007, Project Management Institute - PMI, Chapters Brasileiros. Disponível em: 〈http://www.pmi.org.br〉. Acesso em: 02 mai. 2008.

DUCLÓS, L. C. Simulation cost model for the life-cycle of the software product: a quality assurance approach. 1982. 251 f. Tese (Doutorado em Computer Applications in Industrial and Systems Engineering) USC - University of Southern California. Los Angeles. California. USA.

FORD MOTOR COMPANY. - Potential failure mode and effects analysis (FMEA) Reference Manual. 1988.

FRANCESCHINI, F.; GALETTO, M. A new approach for evaluation of risk priorities of failure modes in FMEA. International Journal of Production Research. v. 29, n. 13, 1991, p. 2991-3002.

GILCHRIST, W. Modeling failure modes and effects analysis. International Journal of Quality \& Reliability Management. v. 10, n. 5, 1993, p. 16-23.

cross ref

HE, S. et al. A new approach to performance analysis of ejector refrigeration system using Grey system theory. Journal Applied Thermal Engineering. Shanghai Jiao Tong University. Shanghai. 2008.

HELDMAN, K. Gerência de projetos: Guia para o exame oficial do PMI. 5. ed. Rio de Janeiro: Campus, 2006.

KEMA. International standard for ISO 13488, KEMA Co. 1996.

KERZNER, H. Gestão de projetos: as melhores práticas. 2. ed. São Paulo: Bookman, 2005.

Mercado Brasileiro de Software. Panorama e tendências. Disponível em: <http://www.abes.org.br/arquivos/MercadoBR-2009-ResumoExec.pdf〉. Acesso em: Abr. 2009.

LEEPHAKPREEDA, T. Grey prediction on indoor comfort temperature for HVAC systems. Journal Expert Systems with Applications. Thammasat University. Thailand. v. 34, 2008, p. 2284-2289.

PALADY, P. FMEA Análise dos Modos de Falha e Efeitos. 3. ed. São Paulo: IMAM, 2004.

PMBOK - Project Management Institute. A guide to the project management body of knowledge. 4. ed. Newton Square, PA, US: Project Management Institute (PMI). 2008. 
PMI. Project Management Institute. Disponível em: <http://www.pmi.org/AboutUs/Pages/Articles-ofInterest.aspx\#project> . Acesso em: 27 Ago. 2008.

PORTER, M. E. Competição: estratégias competitivas essenciais. 13. ed. Rio de Janeiro: Campus, 1999.

PRAHALAD, C. K. A Competência essencial. HSM Management. p.6-11, Mar-Abr., 1997.

PRICE WATERHOUSE COOPERS AUDITORES. Fusões e aquisições no Brasil. 2009. Disponível em: <http://www.pwc.com/pt_BR/br/estudos-pesquisas/assets/rel-fusoes-aquisicoes-mai-09.pdf>. Acesso em: 01 Jun. 2009.

PRITCHARD, C. L. Risk management, concepts and guidance. 3. ed., Virginia, ESI International, 2005.

PUENTE, J. et al. A decision support system for applying failure mode and effects analysis. International Journal of Quality \& Reliability Management. v. 19, n 2, 2002, p. 137-150.

cross ${ }^{\text {ref }}$

SCHUYLER, J. Risk and decision analysis in projects. Project Management Institute, 2 $2^{\mathrm{a}}$ ed. 2001.

THE STANDISH GROUP. Chaos report. 2004. Disponível em: <http://www.standishgroup.com>. Acesso em: 02 ago. 2008.

VANDENBRANDE, W. W. How to use FMEA to reduce the size of your quality toolbox. Quality Progress. v. 31, n.11,1998, p.1822-1835.

VICENTINO, C. História geral. São Paulo: Scipione, 1997.

WANG, C; HSU, L. Using genetic algorithms Grey theory to forecast high technology industrial output. Applied Mathematics and Computation. n.195, 2008, p. 256-263.

WEI, C. H.; CHUNG, M. C. Grey statistics method of technologi selecton for adnanced public transportation systems - The experience of Taiwan. National Science Council of Taiwan. v. 27, n.2, 2003.

WIDEMAN, R. M. Project and program risk management: a guide to managing project risks and opportunities. Upper Darby (USA): Project Management Institute, 1992.

WU, H. H. A comparative study of using Grey relational analysis in multiple attribute decision making problems. Quality Engineering. v. 159, n.2, 2002, p 209-217.

crossef

YIN, R. K. Estudo de Caso: Planejamento e métodos. 3. ed., Porto Alegre: Bookman, 2005. 
APÊNDICE A - LEVANTAMENTO DE RISCOS - DADOS COMPLETOS - ORGANIZADOS PELO METODO FMEA (GR)

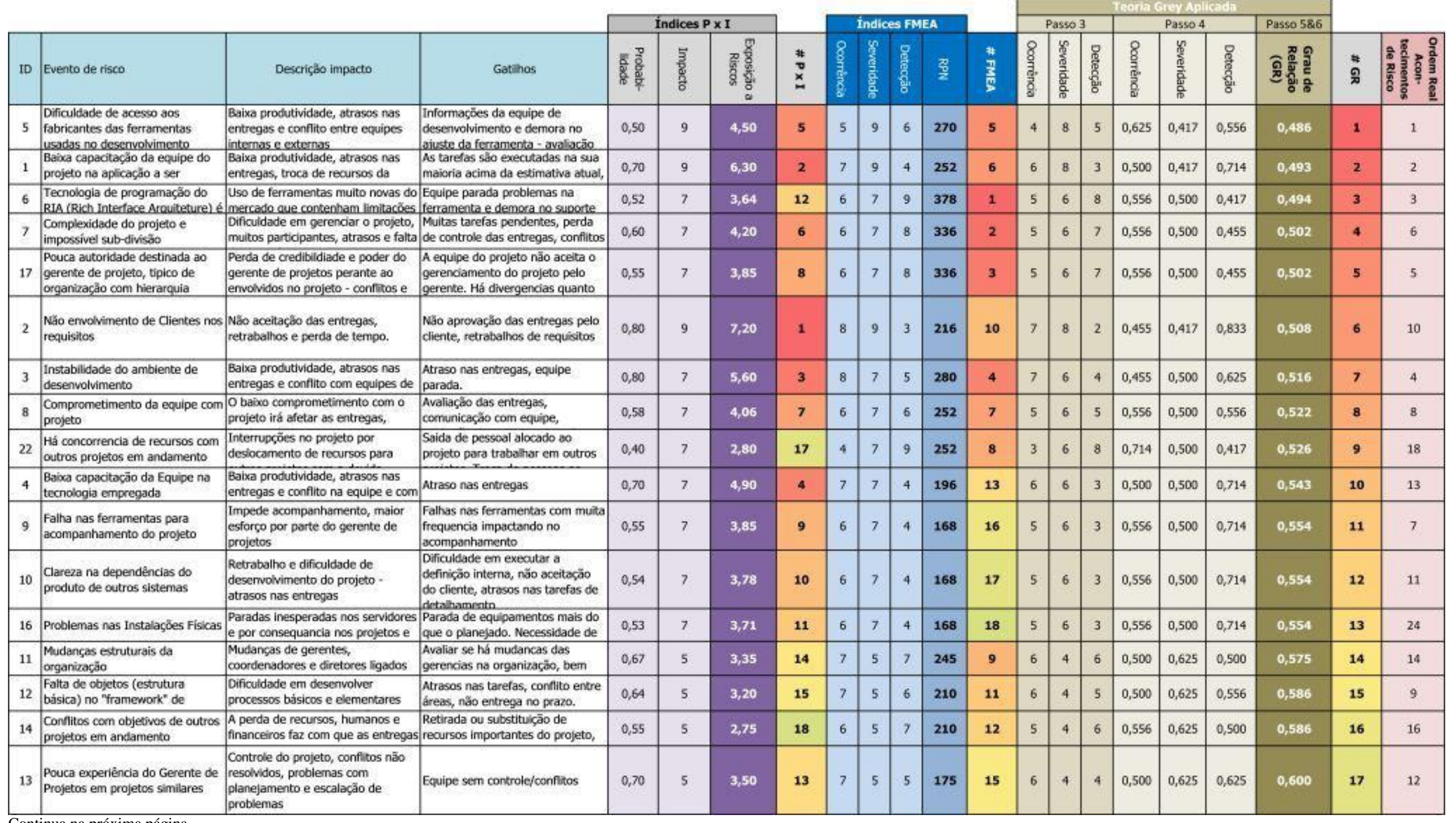

Continua na próxima página.. 


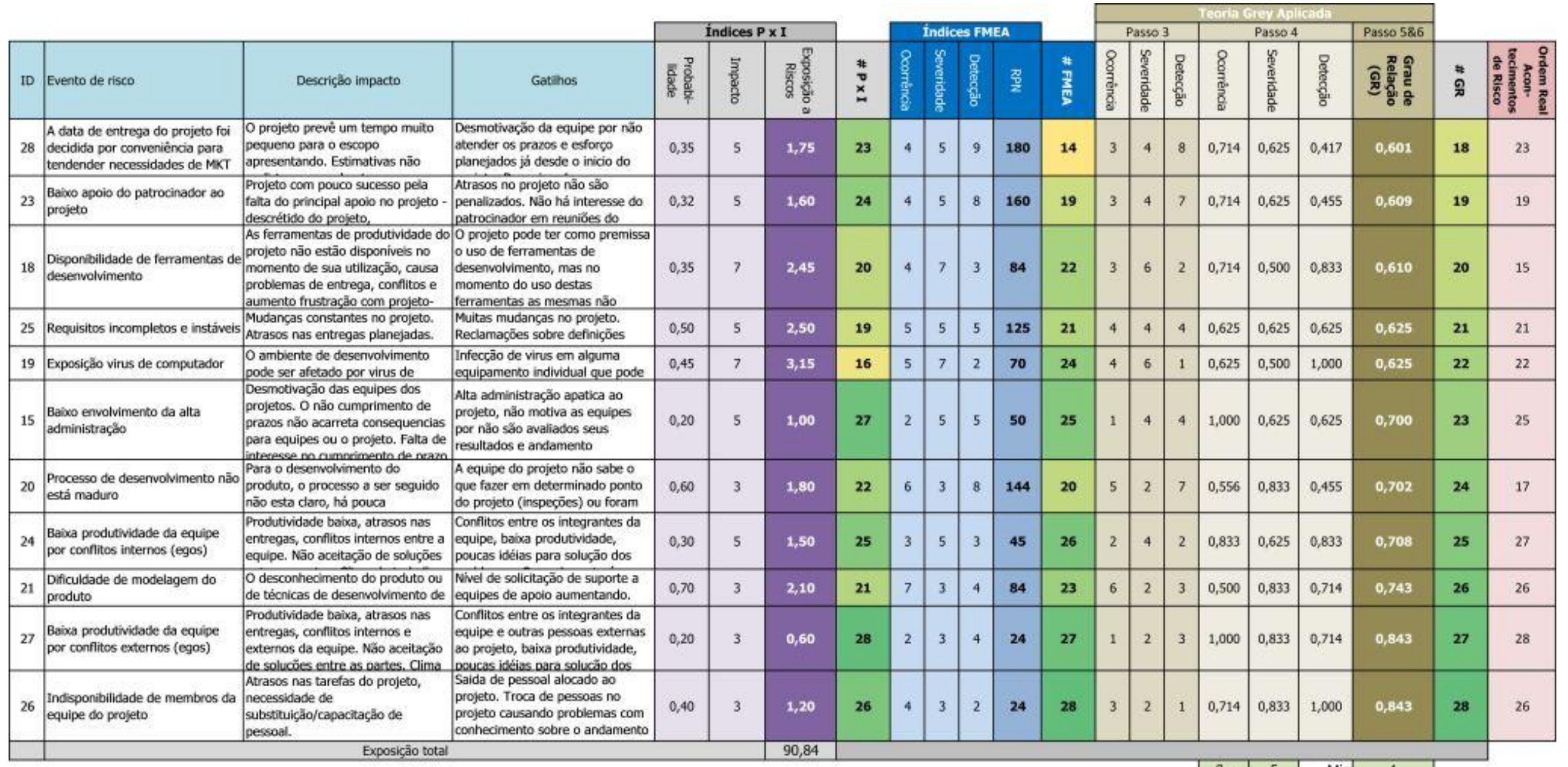

Exposiçāo total 


\section{Dados dos autores:}

Nome completo: Wesley Vieira da Silva

Filiação institucional: Pontifícia Universidade Católica do Paraná

Departamento: Administração de Empresas

Função ou cargo ocupado: Professor do Programa de Pós-Graduação Stricto Sensu em

Administração da PUCPR.

Endereço completo para correspondência (bairro, cidade, estado, país e CEP):

Telefones para contato: (41) 3271-1476 ou (41) 3271-1634

e-mail: wesley.vieira@pucpr.br

Nome completo: Jansen Maia Del Corso

Filiação institucional: Pontifícia Universidade Católica do Paraná

Departamento: Administração de Empresas

Função ou cargo ocupado: Professor do Programa de Pós-Graduação Stricto Sensu em Administração da PUCPR.

Endereço completo para correspondência (bairro, cidade, estado, país e CEP):

Telefones para contato: (41) 3271-1476 ou (41) 3271-1634

e-mail:del.corso@pucpr.br

\section{Nome completo: Roberto Carlos Bonanomi}

Filiação institucional: Pontifícia Universidade Católica do Paraná

Departamento: Administração de Empresas

Função ou cargo ocupado: Professor do Programa de Pós-Graduação Lato Sensu em Gerenciamento de Projetos da PUCPR.

Endereço completo para correspondência (bairro, cidade, estado, país e CEP):

Telefones para contato: (41) 3271-1476 ou (41) 3271-1634

e-mail: roberto.bonanomi@totvs.com.br

Nome completo: Luiz Carlos Duclós

Filiação institucional: Pontifícia Universidade Católica do Paraná

Departamento: Administração de Empresas

Função ou cargo ocupado: Professor do Programa de Pós-Graduação Stricto Sensu em Administração da PUCPR. 
Endereço completo para correspondência (bairro, cidade, estado, país e CEP): Rua Imaculada Conceição, 1155, Bloco Acadêmico, Mestrado/Doutorado em Administração, Curitiba-PR.

Telefones para contato: (41) 3271-1476 ou (41) 3271-1634

e-mail:luiz.duclos@pucpr.br

Recebido para publicação em: 25/09/2010

Aceito para publicação em: 28/11/2010 